\title{
Mass Drug Administration (MDA) for Lymphatic Filariasis Elimination in Uttar Pradesh, India: Issues, Gaps \& Challenges
}

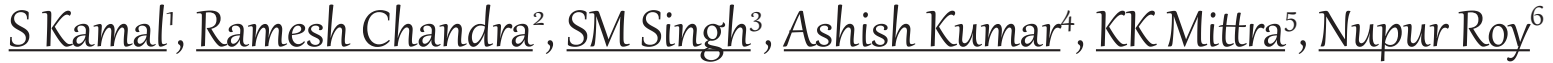 \\ 1,2,3,4,5Regional Office of Health \& Family Welfare (NVBDCP), Kendriya Bhawan, 9th Floor, Aliganj, Lucknow, U.P., India. \\ ${ }^{6}$ Directorate of National Vector Borne Disease Control Programme (NVBDCP), 22, Sham Nath Marg, Delhi, India. \\ DOI: https://doi.org/10.24321/0019.5138.202033
}

\section{I $\quad \mathbf{N} \quad \mathbf{F} \quad \mathbf{O}$}

\section{Corresponding Author:}

$S$ Kamal, Regional Office of Health \& Family Welfare (NVBDCP), Kendriya Bhawan, 9th Floor, Aliganj, Lucknow, U.P., India.

E-mail Id:

shaukatkamal25@yahoo.in

Orcid Id:

https://orcid.org/0000-0002-3358-1572

How to cite this article:

Kamal S, Chandra R, Singh SM, Kumar A, Mittra KK, Roy N. Mass Drug Administration (MDA) for Lymphatic Filariasis Elimination in Uttar Pradesh, India: Issues, Gaps \& Challenges. J Commun Dis 2020; 52(3): 62-73.

Date of Submission: 2020-08-06

Date of Acceptance: 2020-09-30

\section{$\begin{array}{llllllll}\mathbf{A} & \mathbf{B} & \mathbf{S} & \mathbf{T} & \mathbf{R} & \mathbf{A} & \mathbf{C} & \mathbf{T}\end{array}$}

Uttar Pradesh is the largest state of India and is comprised of 75 revenue districts. 51 districts of eastern Uttar Pradesh are endemic for LF and are under Elimination of Lymphatic Filariasis (ELF). In order to ensure elimination of the disease from the people, the mass drug administration (MDA) is being observed in these 51 districts since 2004 by ensuring administration of drug Di-ethyl Carbamazine Citrate (DEC) alone once in a year according to the age excluding children <2years, pregnant women and seriously ill persons. Thereafter, $400 \mathrm{mg}$ albendazole to $>2$ years age group was added with DEC since 2008 . When both the drugs are administered together, the effect is on the adult worm too and therefore the parasite will be destroyed preventing the infected person from developing clinical manifestation. NVBDCP guidelines envisages that administration of drug to the $65 \%$ of the total population or $85 \%$ of the targeted population for five consecutive years will bring down the microfilarimia to $<1 \%$ among the population, ultimately leading to the elimination of the disease after conducting Transmission Assessment Survey (TAS) thrice at an interval of two years among the virgin/protected group of children i.e. 6-7 years. The Transmission Assessment Survey (TAS) is conducted after ensuring $<1 \%$ microfilariamia in 10 additional sites selected randomly.

The state could subject only one district Rampur under post MDA surveillance, which cleared TAS-1 \& TAS-2. The other districts subjected for TAS during 2016, 2017, 2018 \& 2019 but could not qualify for conducting TAS in additional $m$.f. survey as microfilariamia during 2016, 2017 , \& 2018 was found $>1 \%$ and during 2019, the antigenemia was found $>2 \%$ in 13 districts. The possible gaps, issues and challenges at various levels of the programme implementation have been discussed in the present paper, the rectification of which may lead the state for achieving the goal of elimination of the disease.

Keywords: Lymphatic Filariasis, Mass Drug Administration, DEC, Night Blood Survey, Drug Compliance 


\section{Introduction \& Objective}

Lymphatic Filariasis (LF), a vector borne disease transmitted by mosquitoes has been a major public health problem, next to malaria in India. The disease is endemic in 255 districts belonging to 21 states and Union Territories (UT) and is the second highest cause of deformity in human being. In India, National Filaria Control Programme (NFCP) was launched in 1955 for control of bancroftian filariasis with the objectives of undertaking: (i) Delimitation surveys in known endemic areas, (ii) Large scale control measures in selected areas, and (iii) Training of personnel required to man the programme. In two union territories, namely Chhattisgarh and Dadra and Nagar Haveli, no NFCP units has been established till date. The eestimates made on the basis of surveys carried out during the last five decades that about 473 million (348 in rural areas \& 125 in urban areas) people are living in LF endemic areas with a problem magnitude of 23 million disease cases and 31 million people as microfilaria carriers contributing $>70 \%$ LF problem in South East Asia (SEA) region and $38 \%$ of global problem of LF. Uttar Pradesh is the largest state of India and is comprised of 75 revenue districts, of which 112 million (95 million in rural areas \& 17 million in Urban area) people are living in LF endemic areas. Thus, about $24 \%$ people of LF endemic areas of the country inhabit in 51 districts (20\% of the total country's districts) of eastern Uttar Pradesh, with increased density of people in comparison to the country's population. During this period, many advances and researches have compelled the apex level programme personnel and policy makers to think over the problem of common people, who are compelled to bear and live with social stigma among affected people due to irreversible chronic disease manifestations viz. swollen limbs, enlarged scrotum and breast, even chyluria too leading them to economic loss due to confinement on bed for a few days' rest.

The World Health Assembly (WHA) considered Lymphatic Filariasis (LF) as one of the major public health problem and vide its resolution WHA 50.29 resolved to eliminate this disease from the globe by the year 2020. Accordingly, India through its National Health Policy (2002) has also set its goal of Elimination of Lymphatic Filariasis (ELF) in India by 2015 (now 2020). The twin pillars of LF elimination strategy included: 1. Transmission control through Mass Drug Administration (MDA) \& 2. Morbidity Management and Disability Prevention (MMDP).

The transmission control of the disease has been ensured by observing Mass Drug Administration (MDA) in the LF endemic areas. Initially, MDA was launched in 195 districts of the country on $5^{\text {th }}$ June, 2004, in which administration of drug Di-ethyl Carbamazine Citrate (DEC) alone once in a year according to the age excluding children $<2$ years, pregnant women and seriously ill persons was ensured.
Initially, 20 districts of Uttar Pradesh were included and were scaled up subsequently to 51 districts. Six districts of Tamil Nadu and one district of Kerala were subjected for double drug therapy i.e. albendazole along with DEC. The albendazole @ $400 \mathrm{mg}$ to $>2$ years age group was added with DEC since 2008 under MDA programme. After double drug therapy, a third drug, Ivermectin has been added with two drugs according to the height of the individual (According to height, Ivermectin dose schedule is : no drug $<90 \mathrm{cms}$ individuals; $03 \mathrm{mg}$ or 01 tablet to $90-119 \mathrm{cms}$ individuals; $06 \mathrm{mg}$ or 02 tablets to $120-140 \mathrm{cms}$ individuals; $09 \mathrm{mg}$ or 03 tablets to $141-158 \mathrm{cms}$ individuals \& $12 \mathrm{mg}$ or 04 tablets to $>159 \mathrm{cms}$ ) in district Varanasi in February, 2019. The other four districts selected under triple drug therapy included Arwal (Bihar), Shimdega (Jharkhand), Nagpur (Maharashtra) and Yadgir (Karnataka). The number of districts was further increased up to 11 where IDA/MDA was observed during November, 2019. With the collective administration of the drug, the effect is on the adult worm too and, therefore, the parasite would be destroyed preventing the infected person to become a source of infection and from developing clinical manifestation. WHO/ NVBDCP guidelines envisage that administration of drug to the $65 \%$ of the total population or $85 \%$ of the targeted population for five consecutive years will bring down the microfilarimia $<1 \%$ among the population, ultimately leading to the elimination of the disease. This is further assessed by conducting transmission assessment survey (TAS) thrice at an interval of two years among the virgin/protected group of children i.e. 6 to 7 years. The Transmission Assessment Survey (TAS) is conducted after ensuring $<1 \%$ microfilariamia in 10 additional sites selected randomly.

Under the second pillar of the strategy of ELF, lymphodema cases are being imparted training in home based care and management of lymphodema cases and hydrocele operations to be performed at identified $\mathrm{CHCs}$ / District hospitals/Medical colleges in order to alleviate the sufferers and reduce the disease load among the community.

With the implementation of the above strategy, some of the small countries and Indian states/UTs have eliminated the LF disease from their land and nearly 100 Indian districts are progressing towards the elimination of the LF disease, but even after observing MDA since 2004, Uttar Pradesh has only one district Rampur under post MDA surveillance, which cleared TAS- 1 \& TAS-2. The other districts subjected for TAS during 2016, 2017, 2018 \& 2019 but could not qualify for conducting TAS in additional m.f. survey as microfilarimia during 2016, 2017, \& 2018 was found $>1 \%$ and during 2019, the antigenemia was found $>2 \%$ in 13 districts. For any district to be eligible for TAS; (a) It must conduct consecutive five round of MDA, (b) about $65 \%$ of epidemiological coverage, and (c) less than $1 \%$ of $\mathrm{mf}$ rate in both random and sentinel sites. 
This paper contains an In-depth analysis and review of MDA round conducted in Uttar Pradesh since the year 2004 including the data on drug coverage and compliance in each endemic district, $\mathrm{mf}$ rate in sentinel and spot check sites, disease cases (Elephantiasis \& hydroceole). It also includes the findings of Transmission Assessment Surveys (TAS) conducted in the eligible districts and other relevant data which were analysed during this period. The main objective of this study was to find out the possible gaps in programme implementation, various issues and challenges ahead at various levels of the programme implementation. This paper discusses the possible remedies for their rectification, so that the state may make use of them in achieving the goal of elimination of this dreaded disease.

\section{MATERIALS AND METHODS}

Gorakhpur and Varanasi of Uttar Pradesh were among the thirteen (13) districts of the country where pilot project was launched in 1997 to evaluate the efficacy of DEC in reducing $\mathrm{mf}$ load/density in the community through MDA. Based on the encouraging results of the pilot study, the MDA programme was launched in the country in June, 2004, in which 51 districts of UP were also included. The various activities in the state pertaining to the ELF are being coordinated by NVBDCP, Delhi as well as through its Regional Office of Health \& Family Welfare, Lucknow from time to time and necessary monitoring feedback are shared with State Programme Officer (SPO) for further improvement.

The activities conducted in the state were closely observed, monitored and analysed both at state. District, $\mathrm{CHC} / \mathrm{PHC}$ and sub-centre level in order to find out the gaps and lacunae's in succeeding the state in achieving the goal of LF elimination in spite of its best efforts and several rounds of MDA programme since 2004. The possible gaps, issues and challenges at various levels of the programme implementation have been discussed in the present paper, the rectification of which may lead the state for achieving the goal of elimination of the disease set by Government of India.

\section{Results and Discussion}

The coverage of population under MDA in Uttar Pradesh from 2004 to 2018 is reflected in Table-1. It is evident from Table-1 that MDA coverage in terms of drug compliance to total endemic population and targeted population ranged from $19.65 \%$ (2011) to $75.43 \%$ (2015) and $24.43 \%$ (2011) to $89.46 \%$ (2015), respectively. The MDA could not be observed during 2009 due to unavoidable circumstances of the state health authorities. However, the lowest population coverage, against both total \& targeted population was observed in the year 2011, the possible reason to this achievement may be assigned to the least number of districts (14 only) observed MDA but the same thing was reiterated in the year 2016, when only 15 districts observed MDA but the denominator used in this case was changed to the total population of the 15 districts and not the total population of the endemic districts. However, the year of MDA launch (2004) \& 2013 could not achieve the stipulated targets of drug compliance in terms of total LF endemic \& targeted population (Table-3). Thus, as a whole, the state on the basis of this average coverage does not fulfil the criteria as at least continued 05 MDA observance, drug compliance must have been $65 \%$ and $85 \%$ to total LF endemic \& targeted population, respectively and lastly the microfilaria positivity has become $<1 \%$, for proceeding towards next step of elimination of the disease i.e. transmission Assessment Survey TAS), which is conducted thrice at an interval of two years On perusal of district wise drug compliance. Some districts fulfilled the criteria for conducting TAS-1. The parasitological survey carried out prior to the MDA observance has been reflected in Table-2, which shows gradual decline in the microfilaria positivity from 2004 to 2016 but increased thereafter as the close monitoring and cross-check of blood smears was carried out from $\mathrm{ROH}$ \& FW, Lucknow. There is a gradual decline in the disease cases (lymphedema \& hydrocele both) but contrary to the national disease prevalence of gradual increase among the population. On the basis of prevailing data, 18 districts on fulfilling the criteria were proposed for TAS-1 during 2015-16, out of which two districts could not clear the additional microfilaria (m.f.) survey as both reported $>1 \%$ microfilaria positivity. On conducting TAS-1 in 16 districts, only 04 districts could clear TAS-1 \& 12 districts could not clear TAS-1 (Fig.-1\& Table-4). Similarly, during 2016-17, 18 districts of U.P. were proposed to conduct TAS-1, (Fig.-2) and were subjected for Pre-TAS additional m.f. survey, in which none of the district could qualify for conducting TAS-1 due to reporting of $>1 \%$ m.f. rate, still then two districts Hardoi \& Varanasi conducted TAS-1 (Fig.-3). District Varanasi \& Hardoi reported $>7 \%$ \& $10 \%$ filaria antigenemia and could not clear TAS-1. During 2017-18, 03 districts, namely, Azamgarh, Barabanki \& Sonbhadra were proposed for TAS-1 (Fig.-4) but the fate was the same as of preceding districts, as they could not qualify in additional m.f. survey for conducting TAS-1 due to reporting of $>1 \%$ m.f. rate. During 2018-19, 08 districts were proposed for TAS-1 (Fig.5) \& the state decided to conduct filaria antigenemia test with Filaria Test Strip (FTS) in 04 sentinel sites with a test sample size 300 tests from each site. These districts also, could not qualify for conducting TAS-1, as they reflected $>2 \%$ filaria antigenemia. TAS- 2 was also conducted in JulyAugust, 2018 (Table-5), in which two Evaluation Units (EU) of district Rampur could clear TAS-2, whereas 02 EUs of district Chandauli and one EU each of district Etawah \& Kaushambi could not clear TAS-2. During 2019-20, 04 were 
proposed for TAS-1 (Fig.-6) \& to conduct filaria antigenemia test with FTS in 04 sentinel sites with a test sample size 300 tests from each site. These districts too, could not qualify for conducting TAS- 1 , as they reflected $>2 \%$ filaria antigenemia. If, such trend continues, then no district/ area is going to achieve the targets of LF elimination. This led to think seriously on the problem and put forward the gaps, issues with challenges for state to rectify them moderately or completely according to requirement timely, so that the aim can be fulfilled.

Table I.Details of Coverage \& Compliance of population under MDA in Uttar Pradesh from 2004 to 2018

\begin{tabular}{|c|c|c|c|c|c|c|c|}
\hline $\begin{array}{c}\text { S. } \\
\text { No. }\end{array}$ & Year & $\begin{array}{c}\text { No. Of LF } \\
\text { endemic } \\
\text { district } \\
\text { covered }\end{array}$ & $\begin{array}{c}\text { Total } \\
\text { population } \\
\text { at risk }\end{array}$ & $\begin{array}{c}\text { Eligible } \\
\text { Population }\end{array}$ & $\begin{array}{c}\text { Covered } \\
\text { Population }\end{array}$ & $\begin{array}{c}\text { \% Drug Compliance } \\
\text { coverage against } \\
\text { eligible population }\end{array}$ & $\begin{array}{c}\text { \% Drug } \\
\text { Compliance } \\
\text { coverage against } \\
\text { total population }\end{array}$ \\
\hline 1. & 2004 & 20 & 66095557 & 58171536 & 38628588 & 66.40 & 58.44 \\
\hline 2. & 2005 & 50 & 129098055 & 113359584 & 80524028 & 71.03 & 62.37 \\
\hline 3. & 2006 & 50 & 131977848 & 115673236 & 87880300 & 75.97 & 66.59 \\
\hline 4. & 2007 & 50 & 136400266 & 117409513 & 93775777 & 79.87 & 68.75 \\
\hline 5. & 2008 & 50 & 136056859 & 117547788 & 97979211 & 83.35 & 72.01 \\
\hline 6. & 2009 & 0 & 138404387 & 119965504 & & Not Observed & \\
\hline 7. & 2010 & 50 & 138605680 & 121884012 & 98337004 & 80.68 & 70.95 \\
\hline 8. & 2011 & 14 & 137422992 & 33572698 & 27010429 & 24.43 & 19.65 \\
\hline 9. & 2012 & 51 & 140184041 & 118989824 & 98940253 & 83.15 & 70.58 \\
\hline 10. & 2013 & 51 & 145515968 & 121034492 & 85554741 & 70.69 & 58.79 \\
\hline 11. & 2014 & 51 & 147512101 & 124345787 & 103989192 & 83.63 & 70.50 \\
\hline 12. & 2015 & 33 & 98227893 & 82823361 & 74096929 & 89.46 & 75.43 \\
\hline 13. & 2016 & 15 & 35852778 & 30218783 & 25571132 & 84.62 & 71.32 \\
\hline 14. & 2017 & 47 & 144681937 & 122894557 & 106152809 & 86.38 & 73.37 \\
\hline 15. & 2018 & 50 & 148091728 & 126289182 & 105129927 & 83.25 & 70.99 \\
\hline
\end{tabular}

Table 2.The data showing Parasitological findings under ELF in Uttar Pradesh from 2004 to 2018

\begin{tabular}{|c|c|c|c|c|c|c|c|c|c|c|c|c|}
\hline \multirow{3}{*}{$\begin{array}{r}\text { SI. } \\
\text { No. }\end{array}$} & \multirow[b]{3}{*}{ Year } & \multirow{3}{*}{$\begin{array}{c}\text { No. Of } \\
\text { LF } \\
\text { endemic } \\
\text { district } \\
\text { covered }\end{array}$} & \multicolumn{6}{|c|}{ Night Blood Survey (NBS) } & \multirow[b]{3}{*}{$\begin{array}{l}\text { m.f. } \\
\text { Rate } \\
(\%)\end{array}$} & \multirow{3}{*}{$\begin{array}{c}\text { No. of } \\
\text { Lymphode } \\
\text { ma Cases }\end{array}$} & \multirow{3}{*}{$\begin{array}{c}\text { No. Of } \\
\text { Hydrocele } \\
\text { Cases }\end{array}$} & \multirow{3}{*}{$\begin{array}{l}\text { Total } \\
\text { Filaria } \\
\text { Cases }\end{array}$} \\
\hline & & & \multicolumn{2}{|c|}{ SENTINEL } & \multicolumn{2}{|c|}{ SPOT CHECK } & \multicolumn{2}{|c|}{ TOTAL } & & & & \\
\hline & & & $\begin{array}{l}\text { No. Of B/S } \\
\text { Collected/ } \\
\text { Examined }\end{array}$ & $\begin{array}{c}\text { No. Of B/S } \\
\text { m.f. } \\
\text { Positive }\end{array}$ & $\begin{array}{l}\text { No. Of B/S } \\
\text { Collected/ } \\
\text { Examined }\end{array}$ & $\begin{array}{c}\text { No. Of B/S } \\
\text { m.f. } \\
\text { Positive }\end{array}$ & $\begin{array}{l}\text { No. Of B/S } \\
\text { Collected/ } \\
\text { Examined }\end{array}$ & $\begin{array}{c}\text { No. Of } \\
\text { B/S m.f. } \\
\text { Positive }\end{array}$ & & & & \\
\hline 1 & 2 & 3 & 9 & 10 & 11 & 12 & 13 & 14 & 15 & 16 & 17 & 18 \\
\hline 1 & 2004 & 20 & & & & & 8704 & 163 & 1.87 & & & 0 \\
\hline 2 & 2005 & 50 & & & & & 147223 & 1407 & 0.96 & 101049 & 45749 & 146798 \\
\hline 3 & 2006 & 50 & 99633 & 835 & 81029 & 654 & 180662 & 1489 & 0.82 & 101272 & 41217 & 142489 \\
\hline 4 & 2007 & 50 & 96894 & 462 & 94148 & 387 & 191042 & 849 & 0.44 & 106008 & 41093 & 147101 \\
\hline 5 & 2008 & 50 & 98216 & 376 & 91136 & 397 & 189352 & 773 & 0.41 & 80312 & 37671 & 117983 \\
\hline 6 & 2009 & 0 & 0 & 0 & 0 & 0 & 0 & 0 & 0.00 & & & 0 \\
\hline 7 & 2010 & 50 & 100832 & 345 & 95006 & 252 & 195838 & 597 & 0.30 & & & 0 \\
\hline 8 & 2011 & 14 & & & & & 52190 & 127 & 0.24 & 22559 & 9378 & 31937 \\
\hline 9 & 2012 & 51 & 96638 & 0 & 98046 & 0 & 194684 & 665 & 0.34 & 88488 & 31533 & 120021 \\
\hline 10 & 2013 & 51 & 98683 & 174 & 99925 & 183 & 198608 & 357 & 0.18 & & 26533 & 26533 \\
\hline 11 & 2014 & 51 & 100012 & 143 & 99490 & 192 & 199502 & 335 & 0.17 & 91729 & 27406 & 119135 \\
\hline 12 & 2015 & 33 & 65076 & 35 & 63747 & 63 & 128823 & 98 & 0.08 & 77691 & 19000 & 96691 \\
\hline 13 & 2016 & 15 & 24243 & 19 & 24012 & 64 & 48255 & 83 & 0.17 & 11562 & 4315 & 15877 \\
\hline 14 & 2017 & 47 & 93586 & 467 & 94083 & 459 & 187669 & 926 & 0.49 & 97898 & 25895 & 123793 \\
\hline 15 & 2018 & 50 & 99007 & 462 & 98845 & 447 & 197852 & 909 & 0.92 & 93952 & 26401 & 120353 \\
\hline
\end{tabular}


Table 3.Some Specific observations pertaining to MDA coverage in Uttar Pradesh from 2004 to 2018

\begin{tabular}{|c|c|c|}
\hline S. No. & Year of MDA & Specific Observations \\
\hline 1. & 2004 & District Pratapgarh was only one district to achieve $>90 \%$ coverage of targeted population \\
\hline 2. & 2005 & 23 distts. With $<71 \%$ coverage \\
\hline 3. & 2006 & 16 distts. With $<76 \%$ coverage \\
\hline 4. & 2007 & 18 distts. With $<80 \%$ coverage \\
\hline 5. & 2008 & 16 distts. With $<83 \%$ coverage \\
\hline 6. & 2009 & MDA Not Observed \\
\hline 7. & 2010 & 27distts. With $<85 \%$ coverage \\
\hline 8. & 2011 & 50\% distts. With $<80 \%$ coverage \\
\hline 9. & 2012 & 09distts. With $<80 \%$ coverage \\
\hline 10. & 2013 & 13distts. With $<80 \%$ coverage \\
\hline 11. & 2014 & 03distts.9shahjahanpur,Sultanpur \& St.Ravidas Nagar) With $<80 \%$ coverage \\
\hline 12. & 2015 & District Sant Ravi Das Nagar Bhadohi with $<80 \%$ coverage of eligible .Population \\
\hline 13. & 2016 & District Auraiya, Allahabad, Jalaun, Basti \& SPN with <80\% coverage of eligible .Population. \\
\hline 14. & 2017 & $\begin{array}{c}\text { District Auraiya, Allahabad, Chitrakoot, Jalaun \& Kheri with }<80 \% \text { coverage of eligible } \\
\text {.Population }\end{array}$ \\
\hline 15. & 2018 & $\begin{array}{c}\text { District Chandauli, Chitrakoot, Fatehpur, Ghazipur, Jalaun, Lucknow, Mirzapur, Raebareli, } \\
\text { Shravasti, Sonbhadra with <80\% coverage. } m . f . \text { rate almost equal in sentinel \& spot check } \\
\text { sites }\end{array}$ \\
\hline
\end{tabular}

Table 4.Evaluation Unit (EU) wise findings of TAS-I conducted in Uttar Pradesh during 2015-16

\begin{tabular}{|c|c|c|c|c|c|c|c|c|c|c|c|c|c|c|c|c|c|c|c|c|}
\hline & Assessment surveys & s (mapping, senti & inel sites, sp: & otcheck: & ites, and & TAS) & & MAF & & & & & & & $\mathrm{Ag} / \mathrm{Ab}$ & & & & & \\
\hline & $\begin{array}{l}\text { Name of evaluation unit } \\
\text { for TAS only) }\end{array}$ & $\begin{array}{l}\text { Name of } \\
\text { implementation } \\
\text { unit }\end{array}$ & $\begin{array}{l}\text { Name of } \\
\text { survey site }\end{array}$ & $\begin{array}{l}\text { Date of } \\
\text { suney } \\
\text { (month) }\end{array}$ & $\begin{array}{l}\text { Date of } \\
\text { the first } \\
\text { round of } \\
\text { MOA } \\
\text { (year) }\end{array}$ & $\begin{array}{c}\text { Number } \\
\text { of MOA } \\
\text { rounds } \\
\text { comple } \\
\text { ted }\end{array}$ & $\mid \begin{array}{c}\text { Numbe } \\
\text { rot } \\
\text { people } \\
\text { examin } \\
\text { ed }\end{array}$ & $\begin{array}{c}\text { Numb } \\
\text { or of } \\
\text { people } \\
\text { positiv } \\
-\end{array}$ & $\%$ & \begin{tabular}{|c|} 
Dirg \\
nosti \\
$c$ \\
test
\end{tabular} & $\begin{array}{c}\text { Age } \\
\text { tange }\end{array}$ & $\begin{array}{c}\text { Survey } \\
\text { site }\end{array}$ & $\begin{array}{c}\text { \# schoois } \\
\text { or } E A \\
\text { targoted }\end{array}$ & $\begin{array}{l}\text { Targot } \\
\text { sample } \\
\text { size }\end{array}$ & $\begin{array}{c}\text { Number of } \\
\text { people } \\
\text { examined }\end{array}$ & $\begin{array}{c}\text { Number } \\
\text { of } \\
\text { people } \\
\text { positive }\end{array}$ & positive & $\begin{array}{c}\text { Number } \\
\text { of imvalid } \\
\text { teasts }\end{array}$ & $\begin{array}{l}\text { Critical } \\
\text { cut-off }\end{array}$ & Decision \\
\hline 1 & Auraiya EU & Auraiya & Auralya & Mar: 16 & 2005 & 9 & 5032 & 1 & 0.02 & FTS & 6-7years & school & 46 & 1692 & 1699 & 159 & 9.36 & 71 & 20 & Fail \\
\hline 2 & Chitrakoot EU & Chitrakoot & Chitrakoot & Mar-16 & 2005 & 9 & 5060 & 0 & 0.00 & FTS & 6-7years & school & 43 & 1684 & 1775 & 207 & 11.66 & 73 & 20 & Fait \\
\hline 3 & Etawah EU & Etawah & Etawah & Mar-16 & 2005 & 9 & 5008 & 0 & 0.00 & FTS & 6-7years & school & 42 & 1692 & 1699 & 15 & 0.88 & 2 & 20 & Pass \\
\hline 4 & Jalasin EU & Jalaun & Jalaun & Mar-16 & 2005 & 9 & 5080 & 6 & 0.12 & FTS & 6-7years & school & 53 & 1692 & 1692 & 148 & 8.75 & 30 & 20 & Fait \\
\hline 5 & Kaustharnbhi EU & Kaushambhi & couverembti & Mar-16 & 2005 & 9 & 5000 & 0 & 0.00 & FTS & 6.7years & school & 30 & 1692 & 2026 & 5 & 0.25 & 22 & 20 & Pass \\
\hline 6 & Mahoba EU & Mahoba & Mahoba & Mar-16 & 2005 & 9 & 5011 & 0 & 0.00 & FTs & 6-7years & school & 41 & 1556 & 1679 & 129 & 7.68 & 42 & 20 & Fail \\
\hline 7 & Ambedkarnagar EU 1 & Ambedkar nagar & 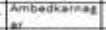 & Mar-16 & 2005 & 10 & 5136 & 5 & 0.10 & FTS & 6-7years & school & 40 & 2692 & 744 & 56 & 7.53 & 93 & 20 & contd. \\
\hline 8 & Ambedkarnogar EU 2 & Ambedkar nagar & Ambedtamants & Mar-16 & 2005 & 10 & 5136 & 5 & 0.10 & FTS & 6-7years & school & 33 & 1692 & 1254 & 109 & 8.69 & 99 & 20 & Contd. \\
\hline 9 & Ballia EU1 & Ballia & Ballia & Mar:16 & 2004 & 11 & 4944 & 2 & 0.04 & ICT & 6-7years & schiool & 32 & 1692 & 1810 & 109 & 6.02 & 19 & 20 & Fail \\
\hline 10 & Ballia EU 2 & Ballia & Balliá & Mar-16 & 2004 & 11 & 4944 & 2 & 0.04 & ICT & 6-7years & school & 31 & 1692 & 1826 & 103 & 5.64 & 49 & 20 & Faii \\
\hline 11 & Basti EU1 & Basti & Basti & Mar-16 & 2004 & 11 & 5147 & 7 & 0.14 & FTS & 6-7years & school & 37 & 1692 & 184 & 39 & 2.12 & 150 & 20 & Fail \\
\hline 12 & Basti EUz & Basti & Basti & Mar-16 & 2004 & 11 & 5147 & 7 & 0.14 & FTS & 6-7years & school & 37 & 1692 & 1904 & 33 & 1.73 & 210 & 20 & contd. \\
\hline$\frac{12}{13}$ & Chandarati EU 1 & Chandauli & Chandauli & Mar-16 & 2005 & $\frac{4}{9}$ & 5151 & 0 & 0.00 & FTS & 6-7years & school & 31 & 168 & & 16 & & 6 & 20 & Pass \\
\hline 14 & Chandaull EU 2 & Chandauli & Chandauli & Mar-1 & & 9 & 5151 & 0 & & FTS & 6-7years & school & 3 & & & 1 & & & & \\
\hline 15 & Ghazipur EU 1 & Ghazipur & Ghazipur & Mar-16 & 2004 & 10 & 5039 & 4 & 0.08 & ICT & 6-7years & school & 42 & 1692 & 2512 & 64 & 2.55 & 68 & 20 & Faii \\
\hline 16 & Ghazipur EU 2 & Ghazipur & Ghazipur & Mar-16 & 2004 & 10 & 5039 & 4 & 0.08 & ICT & 6-7years & school & 33 & 1692 & 1948 & 35 & 1.80 & 27 & 20 & Fail \\
\hline 17 & Kushinagar Eu 1 & Kustinagar & \begin{tabular}{|l|} 
Kushinaga \\
$r$
\end{tabular} & Mar-16 & 2004 & 11 & 5043 & 4 & 0.08 & FTS & 6-7years & school & 30 & 1692 & 1665 & 93 & 5.59 & 37 & 20 & $\begin{array}{c}\text { Fail } \\
\text { Contd. }\end{array}$ \\
\hline 18 & Kushinagar EU 2 & Kushinagar & Kuschinaga & Mar-16 & 2004 & 11 & 5043 & 4 & 0.08 & FTS & 0-7vears & school & 31 & 1692 & 1986 & 22 & 1.11 & 36 & 20 & contd. \\
\hline 19 & Maharajganj EU 1 & Mahara) Ganj & Maharajga & Mar-16 & 2004 & 11 & 5038 & 0 & 0.00 & FTS & 6-7years & schoot & 39 & 1692 & 1716 & 24 & 1.40 & 81 & 20 & Fail \\
\hline 20 & Maharajganj EU 2 & Maharaj Ganj & 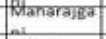 & Mar-16 & 2004 & 11 & 5038 & 0 & 0.00 & FTS & 6-7years & school & 32 & 1692 & 1750 & 57 & 3.26 & 35 & 20 & Fail \\
\hline 21 & hampur EU 1 & Rampur & Rampur & Mar-16 & 2005 & 9 & 5000 & 0 & 0.00 & FTS & 6-7years & schoot & 45 & 1692 & 1399 & 6 & 0.43 & 81 & 20 & contd. \\
\hline 22 & hampur EU 2 & Rampur & Rampur & Mar -16 & 2005 & 9 & 5000 & 0 & 0.00 & FTS & 6-7years & school & 43 & 1692 & 1775 & 16 & 0.90 & 77 & 20 & Pass \\
\hline 23 & Siddharth Nogar EU 1 & siddharthnagar & $\begin{array}{l}\text { Siddharth } \\
\text { Nagar }\end{array}$ & Mar-16 & 2005 & 9 & 5039 & $\circ$ & 0.00 & FTS & 0-7years & school & 31 & 1692 & 1925 & 52 & 2.70 & 494 & 20 & contd. \\
\hline 24 & Siddharth Nogar EU 2 & siddharthnagar & \begin{tabular}{|l|} 
Siddharth \\
Nagar
\end{tabular} & Mar-16 & 2005 & 9 & 5039 & 0 & 0.00 & FTS & 6-7years & school & 32 & 1692 & 1549 & 89 & 5.75 & 173 & 20 & $\begin{array}{c}\text { Fail } \\
\text { contd. }\end{array}$ \\
\hline 25 & Bahraich EU 1 & Bahraich & Bathraich & Mar-16 & 2004 & 11 & 5096 & 6 & 0.12 & FTs & 6-7vears & school & 31 & 1692 & 1836 & 197 & 10.73 & ss & 20 & $\begin{array}{c}\text { Fail } \\
\text { contd. }\end{array}$ \\
\hline 26 & Bahraich EU 2 & Bahraich & Bahraich & Mar 16 & 2004 & 11 & 5096 & 6 & 0.12 & FTS & 6-7years & schoot & 32 & 1692 & 1840 & 365 & 19.84 & 86 & 20 & $\begin{array}{c}\text { Fail } \\
\text { contd. }\end{array}$ \\
\hline 27 & Batraich EU 3 & Batraich & Bathraich & Mar-16 & 2004 & 11 & 5096 & 6 & 0.12 & FTS & 6.7years & school & 31 & 1684 & 1840 & 314 & 17.07 & 46 & C.2olV & tFa! V \\
\hline & TOTAI & & & & & & & & & & & & 978 & 45338 & 46935 & 2475 & 5.27325 & 2198 & 538 & 0 \\
\hline
\end{tabular}


Table 5.Findings of TAS-2 conducted in Uttar Pradesh during 20 I8- 19

\begin{tabular}{|c|c|c|c|c|c|c|c|c|c|c|c|c|}
\hline \multicolumn{13}{|c|}{ Report on Tas-2 Conducted in Schoolclusters from 30 ${ }^{\text {Th }}$ July To $16^{\text {Th }}$ August, 2018 in U.p. } \\
\hline \multirow{2}{*}{$\begin{array}{l}\text { S. } \\
\text { No. }\end{array}$} & \multirow{2}{*}{ District } & \multirow{2}{*}{ EU } & \multirow{2}{*}{$\begin{array}{c}\text { No. of } \\
\text { schools } \\
\text { surveyed }\end{array}$} & \multirow{2}{*}{$\begin{array}{l}\text { Dates of } \\
\text { survey }\end{array}$} & \multicolumn{3}{|c|}{$\begin{array}{l}\text { Total No. of students } \\
\text { present \& tested }\end{array}$} & \multicolumn{4}{|c|}{ Test results } & \multirow{2}{*}{ Remarks* } \\
\hline & & & & & $\begin{array}{l}\text { 1st } \\
\text { ST. }\end{array}$ & $\begin{array}{l}\text { 2nd } \\
\text { ST. }\end{array}$ & Total & $\begin{array}{l}(+) \\
\text { IVE }\end{array}$ & $(-)$ IVE & $\begin{array}{l}\text { INVA } \\
\text {-LID }\end{array}$ & $\begin{array}{l}\text { Cut } \\
\text { off }\end{array}$ & \\
\hline 1. & \multirow{2}{*}{ Rampur } & 1 & 58 & $\begin{array}{c}30^{\text {th }} \text { July to } \\
16^{\text {th }} \text { Aug, } 2018\end{array}$ & 687 & 1099 & 1786 & 09 & 1694 & 83 & 20 & $\mathrm{P}$ \\
\hline 2. & & II & 54 & $\begin{array}{c}30^{\text {th }} \text { July to } 3^{\text {rd }} \\
\text { Aug, } 2018\end{array}$ & 659 & 1117 & 1776 & 11 & 1693 & 72 & 20 & $\mathrm{P}$ \\
\hline 3. & \multirow{2}{*}{ Chandauli } & $\mathrm{N}$ & & $\begin{array}{c}30^{\text {th }} \text { July to } 3^{\text {rd }} \\
\text { Aug, } 2018\end{array}$ & & & 1761 & 150 & 1537 & 74 & 20 & $\mathrm{~F}$ \\
\hline 4. & & $S$ & & $\begin{array}{c}30^{\text {th }} \text { July to } \\
04^{\text {th }} \text { Aug, } 2018\end{array}$ & & & 1685 & 164 & 1394 & 127 & 18 & $\mathrm{~F}$ \\
\hline 5. & Kaushambi & & & $\begin{array}{c}30^{\text {th }} \text { July to } \\
04^{\text {th }} \text { Aug, } 2018\end{array}$ & & & 1716 & 184 & 1509 & 23 & 20 & $\mathrm{~F}$ \\
\hline 6. & Etawah & & & $\begin{array}{c}30^{\text {th }} \text { July to } \\
04^{\text {th }} \text { Aug, } 2018\end{array}$ & & & 1735 & 142 & 1475 & 118 & 20 & $\mathrm{~F}$ \\
\hline
\end{tabular}

*P=Pass \& F==Fail =North \& S=South

Table 6.Data showing details of Human Resources under National Filaria Control Programme (NFCP) in Uttar Pradesh

\begin{tabular}{|c|c|c|c|c|c|c|c|c|c|c|c|c|c|c|c|c|c|c|c|}
\hline \multirow[t]{2}{*}{$\begin{array}{l}\text { S. } \\
\text { No. }\end{array}$} & \multirow[t]{2}{*}{ Name of FCU } & \multicolumn{3}{|c|}{$\begin{array}{l}\text { Filaria } \\
\text { Control } \\
\text { Officer }\end{array}$} & \multicolumn{3}{|c|}{ Biologist } & \multicolumn{3}{|c|}{$\begin{array}{l}\text { Filaria } \\
\text { Inspector }\end{array}$} & \multicolumn{3}{|c|}{$\begin{array}{l}\text { Laboratory } \\
\text { Technician }\end{array}$} & \multicolumn{3}{|c|}{$\begin{array}{l}\text { Insect } \\
\text { Collectors }\end{array}$} & \multicolumn{3}{|c|}{$\begin{array}{l}\text { Sup.Field } \\
\text { Workers }\end{array}$} \\
\hline & & $S$ & $\mathbf{P}$ & V & $S$ & $\mathbf{P}$ & V & $S$ & $\mathbf{P}$ & v & $S$ & $\mathbf{P}$ & $\mathbf{v}$ & $S$ & $\mathbf{P}$ & V & $S$ & $\mathbf{P}$ & v \\
\hline 1 & 2 & 3 & 4 & 5 & 6 & 7 & 8 & 9 & 10 & 11 & 12 & 13 & 14 & 15 & 16 & 17 & 18 & 19 & 20 \\
\hline 1. & Gorakhpur & 1 & 1 & 0 & 0 & 0 & 0 & 6 & 3 & 3 & 2 & 2 & 0 & 4 & 1 & 3 & 8 & 2 & 6 \\
\hline 2. & Deoria & 00 & 00 & 00 & 1 & 1 & 0 & 2 & 2 & 0 & 1 & 1 & 0 & 1 & 1 & 0 & 2 & 0 & 2 \\
\hline 3. & Basti & 00 & 00 & 00 & 1 & 0 & 1 & & 2 & 1 & 2 & 1 & 1 & 4 & 3 & 1 & 2 & 1 & 1 \\
\hline 4. & Azamgarh & 00 & 00 & 00 & 1 & 1 & 0 & 3 & 3 & 0 & 1 & 1 & 0 & 4 & 0 & 4 & 2 & 2 & 0 \\
\hline 5. & Ilia & 00 & 00 & 00 & 1 & 1 & 0 & 3 & 2 & 1 & 2 & 2 & 0 & 4 & 4 & 0 & 2 & 2 & 0 \\
\hline 6. & Mirzapur & 1 & 1 & 0 & 0 & 0 & 0 & 3 & 3 & 0 & 2 & 1 & 1 & 2 & 2 & 0 & 11 & 6 & 5 \\
\hline 7. & Ghazipur & 00 & 00 & 00 & 1 & 0 & 1 & 3 & 2 & 1 & 1 & 1 & 0 & 4 & 1 & 3 & 2 & 2 & 0 \\
\hline 8. & Jaunpur & 1 & 0 & 1 & 0 & 0 & 0 & 3 & 3 & 0 & 1 & 1 & 0 & 4 & 0 & 4 & 3 & 2 & 1 \\
\hline 9. & $\begin{array}{l}\text { Varanasi (Ram } \\
\text { Nagar) }\end{array}$ & 00 & 00 & 00 & 1 & 1 & 0 & 2 & 2 & 0 & 1 & 0 & 1 & 1 & 1 & 0 & 3 & 2 & 1 \\
\hline 10. & Pratapgarh & 00 & 00 & 00 & 1 & 1 & 0 & 2 & 1 & 1 & 1 & 0 & 1 & 1 & 0 & 1 & 2 & 1 & 1 \\
\hline 11. & Fatehpur & 1 & 0 & 1 & 0 & 0 & 0 & 2 & 2 & 0 & 2 & 2 & 0 & 1 & 0 & 1 & 6 & 4 & 2 \\
\hline 12. & Raebareli & 00 & 00 & 00 & 1 & 0 & 1 & 2 & 2 & 0 & 1 & 1 & 0 & 1 & 1 & 0 & 2 & 0 & 2 \\
\hline 13. & Unnao & 00 & 00 & 00 & 1 & 1 & 0 & 2 & 2 & 0 & 1 & 0 & 1 & 1 & 0 & 1 & 2 & 1 & 1 \\
\hline 14. & Lucknow & 1 & 1 & 0 & 0 & 0 & 0 & 3 & 4 & 0 & 1 & 1 & 0 & 4 & 4 & 0 & 2 & 2 & 0 \\
\hline 15. & Hardoi & 00 & 00 & 00 & 1 & 0 & 1 & 2 & 2 & 0 & 1 & 0 & 1 & 1 & 1 & 0 & 2 & 2 & 0 \\
\hline 16. & Sitapur & 00 & 00 & 00 & 1 & 0 & 1 & 2 & 0 & 2 & 1 & 1 & 0 & 2 & 0 & 2 & 4 & 3 & 1 \\
\hline 17. & LakhimpurKheri & 00 & 00 & 00 & 1 & 0 & 1 & 3 & 3 & 0 & 1 & 1 & 0 & 2 & 2 & 0 & 4 & 4 & 0 \\
\hline 18. & Faizabad & 1 & 1 & 0 & 0 & 0 & 0 & 3 & 3 & 0 & 2 & 2 & 0 & 4 & 2 & 2 & 6 & 5 & 1 \\
\hline
\end{tabular}




\begin{tabular}{|c|c|c|c|c|c|c|c|c|c|c|c|c|c|c|c|c|c|c|c|}
\hline 19. & Sultanpur & 00 & 00 & 00 & 1 & 1 & 0 & 2 & 1 & 1 & 1 & 1 & 0 & 1 & 0 & 1 & 1 & 1 & 0 \\
\hline 20. & Gonda & 00 & 00 & 00 & 1 & 1 & 0 & 2 & 2 & 0 & 1 & 1 & 0 & 1 & 1 & 0 & 2 & 0 & 2 \\
\hline 21. & Barabanki & 1 & 1 & 0 & 0 & 0 & 0 & 3 & 3 & 0 & 1 & 1 & 0 & 4 & 2 & 2 & 2 & 2 & 0 \\
\hline 22. & Bahraich & 1 & 1 & 0 & 0 & 0 & 0 & 3 & 3 & 0 & 1 & 0 & 1 & 4 & 3 & 1 & 3 & 2 & 1 \\
\hline 23. & Pilibhit & 00 & 00 & 00 & 1 & 0 & 1 & 3 & 2 & 1 & 1 & 0 & 1 & 2 & 2 & 0 & 4 & 3 & 1 \\
\hline 24. & Shahjahanpur & 00 & 00 & 00 & 1 & 0 & 1 & 5 & 4 & 1 & 1 & 0 & 1 & 4 & 3 & 1 & 8 & 7 & 1 \\
\hline 25. & Rampur & 00 & 00 & 00 & 1 & 0 & 1 & 4 & 4 & 0 & 0 & 0 & 0 & 4 & 3 & 1 & 8 & 8 & 0 \\
\hline 26. & Farrukhabad & 00 & 00 & 00 & 1 & 0 & 1 & 3 & 2 & 1 & 2 & 0 & 2 & 2 & 0 & 2 & 4 & 0 & 4 \\
\hline 27. & Jalaun & 00 & 00 & 00 & 1 & 1 & 0 & 4 & 0 & 4 & 1 & 1 & 0 & 3 & 0 & 3 & 4 & 3 & 1 \\
\hline \multirow{2}{*}{28.} & Banda & 00 & 00 & 00 & 1 & 0 & 1 & 3 & 3 & 0 & 1 & 1 & 0 & 2 & 1 & 1 & 4 & 1 & 3 \\
\hline & Chitrakoot* & 00 & 00 & 00 & 00 & 00 & 00 & 01 & 0 & 01 & 0 & 0 & 0 & 1 & 1 & 0 & 1 & 1 & 0 \\
\hline \multirow[t]{6}{*}{29.} & Hamirpur & 00 & 00 & 00 & 1 & 0 & 1 & 2 & 2 & 0 & 1 & 0 & 1 & 1 & 1 & 0 & 3 & 1 & 2 \\
\hline & Total (A) & 08 & 06 & 02 & 21 & 09 & 12 & 84 & 67 & 17 & 35 & 23 & 12 & 74 & 40 & 34 & 109 & 70 & 39 \\
\hline & Only Clinic & & & & & & & & & & & & & & & & & & \\
\hline & Allahabad & 0 & 0 & 0 & 0 & 0 & 0 & 1 & 1 & 0 & 1 & 1 & 0 & 0 & 0 & 0 & 0 & 0 & 0 \\
\hline & Kanpur Nagar & 0 & 0 & 0 & 0 & 0 & 0 & 1 & 1 & 0 & 1 & 1 & 0 & 0 & 0 & 0 & 0 & 0 & 0 \\
\hline & Total (B) & 0 & 0 & 0 & 0 & 0 & 0 & 2 & 2 & 0 & 2 & 2 & 0 & 0 & 0 & 0 & 0 & 0 & 0 \\
\hline \multicolumn{2}{|c|}{ Grand Total $(A+B)$} & 08 & 06 & 02 & 21 & 09 & 12 & 86 & 69 & 17 & 37 & 25 & 12 & 74 & 40 & 34 & 109 & 70 & 39 \\
\hline
\end{tabular}

\section{$\mathrm{S}=$ Sanctioned, $\mathrm{P}=$ Posted $\& \mathrm{~V}=$ Vacant}

- Officer I/C of FCU: FCO in 8 Units (6 non-medical \& 2 medical), Biologists in 21 units.

- *Banda FCU earlier had its sub-unit at Chitrakoot, needs full strengthening.

- Filaria Control Officer Post in District Lucknow \& Mirzapur are from medical cadre.
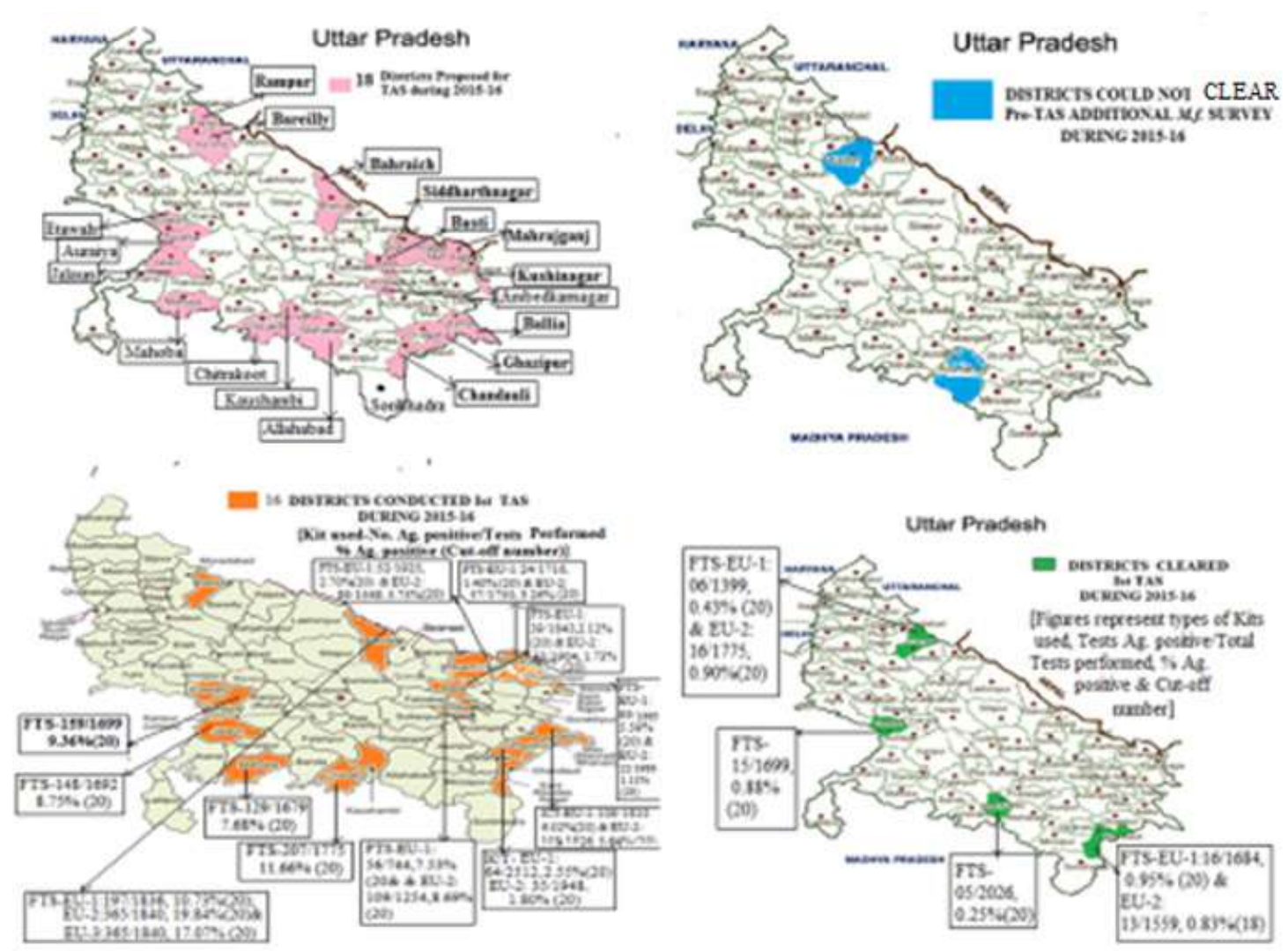

Figure I.I 6 Districts of U.P. subjected for TAS-I during 20 I 5- I6 


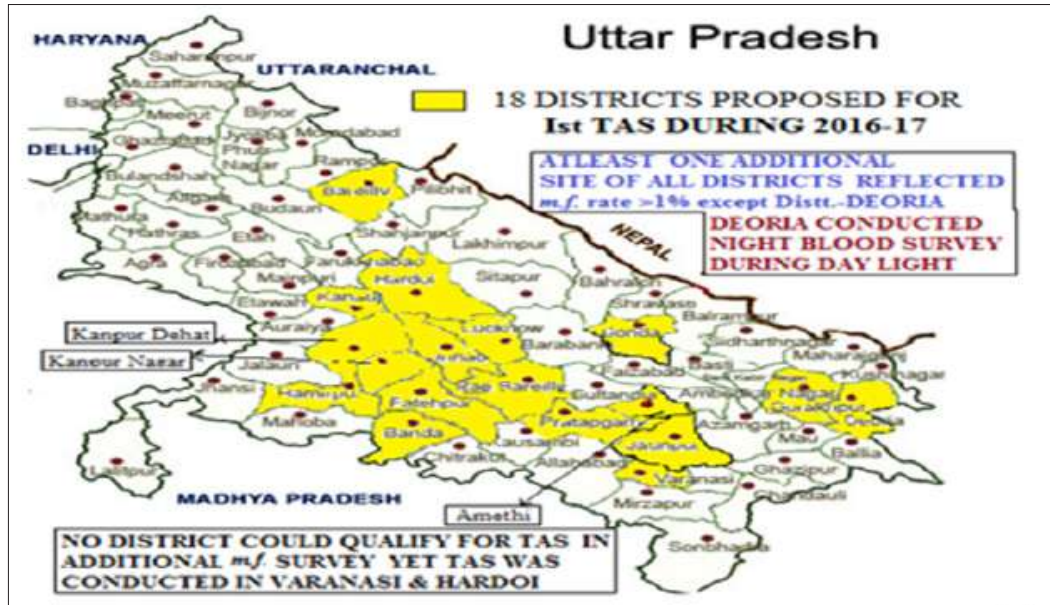

Figure 2. I 8 Districts of U.P. Subjected for Pre-TAS-I additional survey during 20 I 6-I 7

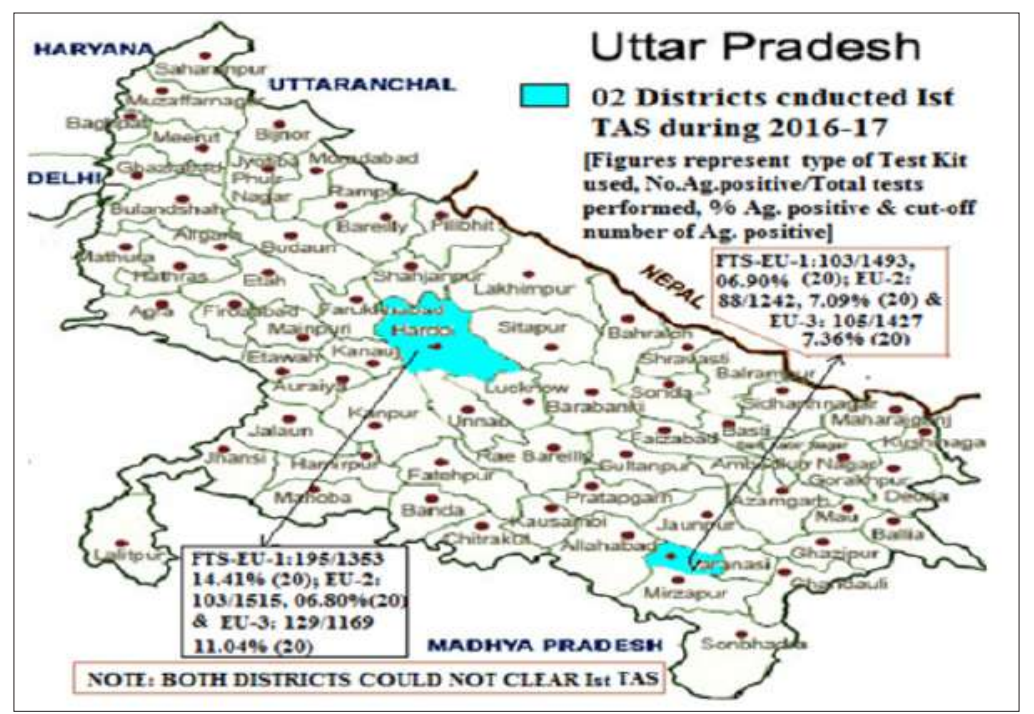

Figure 3.02 Districts of U.P. conducted TAS-I during 2016-I 7

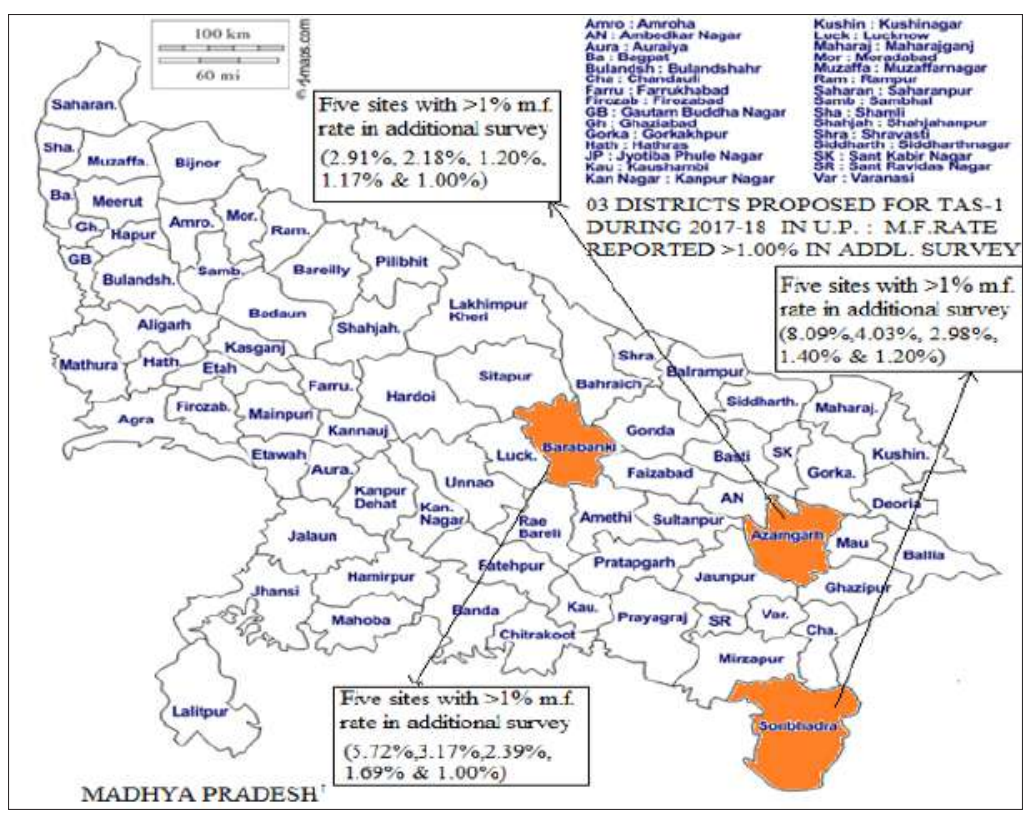

Figure 4.03 Districts of U.P. Subjected for Pre-TAS-I additional mf survey during 20 I 7- 18 


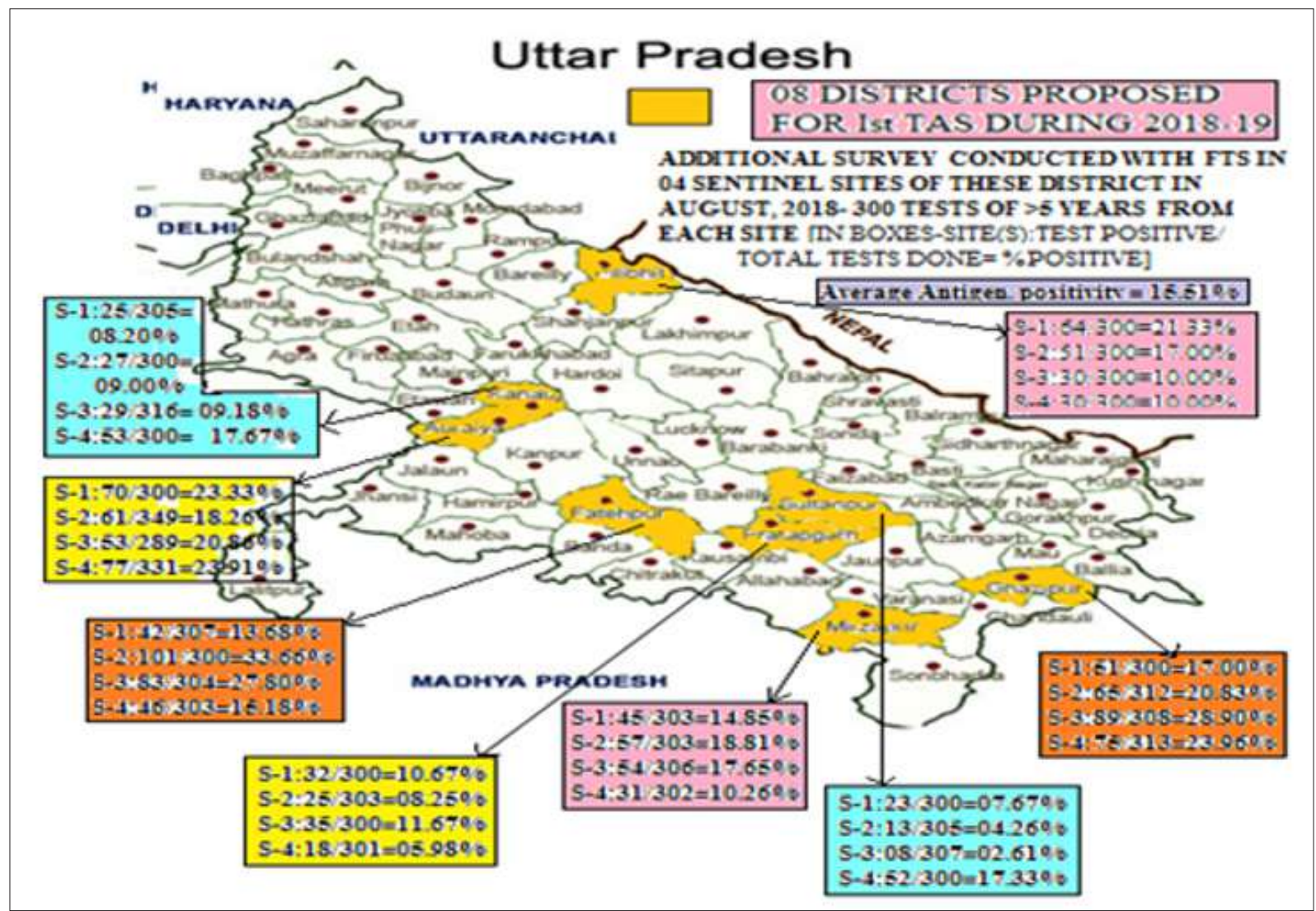

Figure 5.08 Districts of U.P subjected for Pre-TAS-I additional survey during 20 I 8- I 9

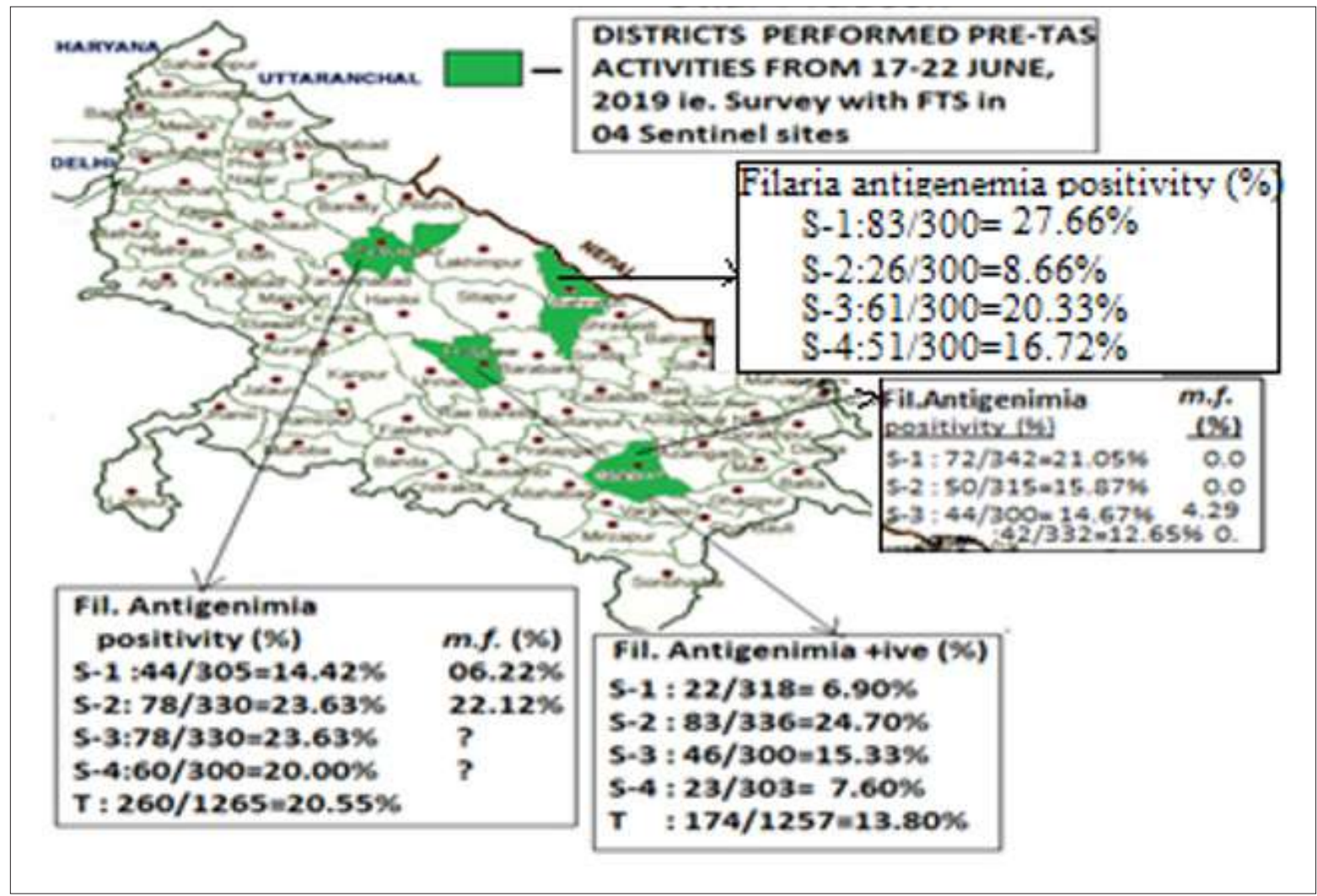

Figure 6.4 Districts of U.P. subjected for Pre-TAS-I additional survey With FTS during 2019-20 


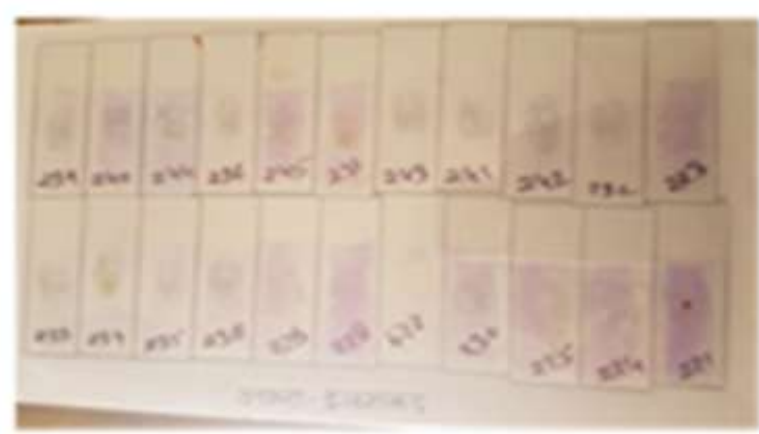

a

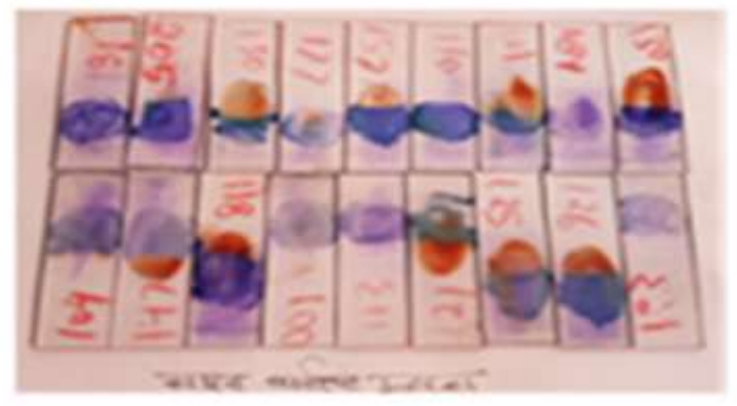

c

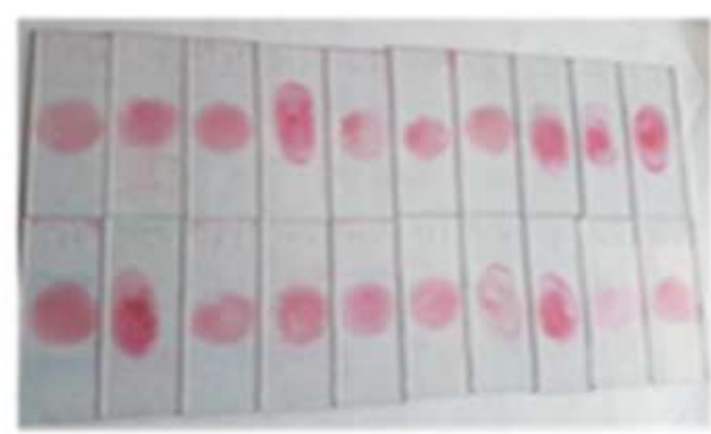

b

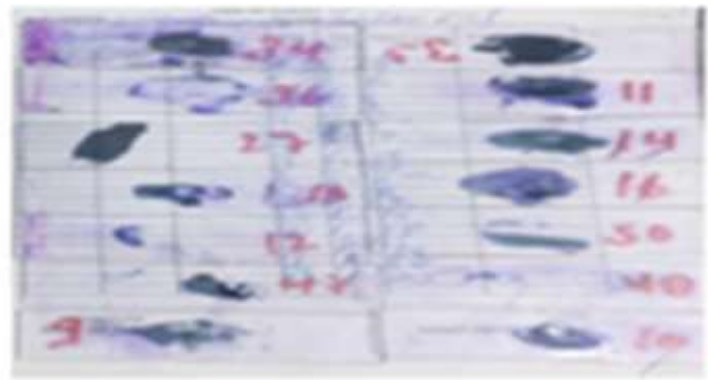

d

Figure 7.Showing different types of blood smears collected in night blood survey in UP during Pre- MDA and Pre-TAS activities. (a) Only thin blood smear with least blood quantity \& poor staining; (b) Artificial color used for preparing smear instead of human blood, (c): The thick blood smears, half stained without de-haemoglobinization; (d) The blood smear not prepared \& stained properly

Further in-depth review and analysis of the data revealed that the various gaps and the issues are leading to malpractices in planning and implementing the programme. The achievement in the coverage of community and quality of the work largely depends on governance of the programme managers together with the capability \& interest of the human resource. The coverage reflected in Table 1 is the average coverage but district wise perusal reflects under coverage to target in many districts (Table 3) and likely PHCs/ Health sub-centres. This is due to the fact that the quality training of drug administrators and supervisors are lacking, which is an important component of MDA programme. The supervision is completely lacking at peripheral grass root level. The convergence and acceptability by the community is lacking due to inadequate and improper IEC activities, which should be round the year irrespective of the date of MDA. The higher level supervision from ROHFW level, verified that the drug administrators at many places have been replaced without any orientation of the new person to the programme. Such practices needs to be avoided. The supervisor is engaged with the other duties, paying least attention to MDA. The possible reason for lack of interest of drug administrator and supervisor may be due to the fact that they did not receive the honorarium from $\mathrm{PHC} /$ district fund managers. This practice is to be abolished by making timely payment to them, for which fund-related official should be made accountable with penalty. Moreover, due to partial coverage with single dose DEC, it is not possible to achieve the goal of elimination of the disease. Can it be achieved by adding additional drugs, is a matter of concern. The single drug DEC is recommended and is being used in the routine programme, proving its efficacy but when it is being administered at mass level, the consumption/ compliance of the drug declines; it is a matter of concern, because it leads to poor coverage and the drug needs no additional combination, if the single drug is consumed in adequate number, for which various technologies are to be practiced to generate the need of the drug among the community. Moreover, keeping in view the half life period of the DEC drug retained in the human body, the schedule of administering the drug DEC may be shifted to evening or bed time, very close to the time of activity of the microfilaria, when they circulate in the peripheral circulatory system, in order to have better effect of the drug.

The self check by the state/district programme manager is performed through the assessment of parasitic load in the community by conducting night blood surveys (NBS) in sentinel and spot check sites. The findings of the NBS conducted (Table 2) prior to MDA observance are not reflecting the true/factual picture of the microfilaria rate as in the districts subjected for Transmission Assessment Survey (TAS) or additional survey prior to TAS, both 
microfilarimia or antegenemia were found much higher. The bare fact attributed to this, is possibly the manpower engaged in conducting the NBS, is non-NFCP oriented, who are deputed for work after short orientation of preparation and examination of blood smears and commit many irregularities in performing the survey viz. many conduct survey (B/S collection) during day time instead of scheduled survey time 20:30 hrs. to 24:00 hrs. Moreover, the blood smears are not prepared in accordance with the guidelines laid down by NVBDCP. At many locations, these slides were neither de-haemoglobinised nor stained properly. Some made smear using artificial colors instead of human blood (Fig. -7). If the survey teams are supervised properly, then they are not supposed to commit such types of irregularities. Moreover, the NFCP staff deployed in the district (Table-6), is least involved in NBS. Further, the NFCP staff is also not conducting the routine surveys and collecting blood smears according to the NFCP guidelines, in which the unit has to collect and examine 1600-1700 blood smears per month ${ }^{2}$ (at least $1500 /$ month). If the NFCP staff is deployed for NBS prior to MDA, the perusal of reports reflected $>1 \%$ microfilaria rate in the area even in the jurisdiction of unit, which was reporting $<1 \%$ microfilaria rate in routine monthly surveys. This indicates that strict supervision from various level is lacking and the staff is not being held accountable. To ensure quality control, there is provision of cross check of blood smear by Regional offices of Health \& family Welfare located in various states, but the blood smears from the state are not being made available on schedule time. In some districts, TAS-1 could not have been effected during 2015-16, if the results of night blood survey (NBS) in additional survey have been made properly but the discrepancy in results of these districts could be detected only when the districts sent blood smear for cross check to ROHFW after conducting TAS-1. The staff of NFCP units (Table-6) in the state, can be deputed for conducting NBS prior to MDA, both in parental proper NFCP unit district and newly created district from it under strict supervision with fixing accountability for non-compliance of proper directives. The factual picture of the microfilaria rate will help not only to undertake focussed activities for drug administration but will prevent undue wastage of resources in conducting the futile exercises of TAS. Govt. of India during 1994 directed the states to create posts of District Filaria Control Officers ${ }^{2}$ to tackle and implement the NFCP properly even in rural areas with high disease prevalence.

Four districts cleared TAS-1 during 2015-16 but except Rampur, three districts, Chandauli, Kaushambi \& Etawah, could not clear TAS-2 conducted during 2018-19 (Table-5). This may possibly be attributed to the fact that there is no anti-vector measure in the rural areas. The NVBDCP guidelines ${ }^{1}$ envisage that the anti-vector measures are to be continued in the NFCP unit areas, which are restricted to urban areas only. The dual standard will not be helpful for th ector measures as strategic pillar, then why filaria disease has been deprived from incorporation of anti-vector measures in rural areas. It is a matter of great concern to all thinking to eliminate the disease either in Uttar Pradesh or in other states. The resurgence of malaria from most of the Indian subcontinent is the evidential instance. Thus, the goal of elimination of filariasis, can be achieved by getting real time picture of the achievements be it the compliance coverage or parasitological surveys; it is a great challenge and can be succeeded by tackling the various issues \& gaps enlighten.

The date of National Filaria Day (NFD) was fixed for conducting MDA at one time, for which time framed activities were chalked out to be followed by states/ UTs, but it has been observed that MDA is not keeping pace with national guidelines. There is no fixed date for conducting MDA. It is largely governed by the availability of fund, availability and procurement of Drugs (DEC) Albendazole/Ivermectin) and local situations viz. floods, elections, holidays etc. Uncertainty for MDA leads to delay in timely preparation and implementation of Micro plan of State and District as per National guidelines. Delay in Programme implementation occurs due to late release of fund/ logistics/ supplies. The delay in submission of SOE/UC by the districts to State and from State to Centre dislocates availability of fund for ELF activities. Many a times States cannot send SOE/UC for non-receipt of the same from district. Lack of proper logistics (good quality microscopes, slides, stains, chemicals \& other supplies) nd mobility support also adversely affect the quality of the work. There is an urgent need for adequate and round the year training with proper orientation to Health, NGO and other supporting partners, continued orientation of state and district level officers by the Central team as per National Guidelines for LF elimination. ${ }^{1,3,4}$ In addition to this, Intensive and sustainable IEC activities (in different forms) at different levels of programme implementation is also needed to make MDA programme more successful and to enhance compliance.

\section{Acknowledgement}

The authors are thankful to the State VBD Programme Officer, U.P., Lucknow for providing the data as well as for the support extended during the field visits to different districts of the state. Thanks are also due to Dr. N. Dhingra, Director, National Vector Borne Disease Control Programme (NVBDCP), Delhi for constant encouragement during the preparation of this manuscript.

\section{Conflicts of Interest: None}

\section{References}

1. Operational Guidelines on Elimination of Lymphatic 
Filariasis, National Vector Borne Disease Control Programme (NVBDCP), Gol publn. pp.87.

2. National Filaria Control Programme Operational Manual, 1995,National Vector Borne Disease Control Programme (NVBDCP), Golpubln. pp.127.

3. Guidelines on Elimination of Lymphatic Filariasis, India, - Interruption of Disability alleviation through home based management of lymphodema and hospital based hydrocelectomy, 2009, National Vector Borne Disease Control Programme (NVBDCP), Gol publn. pp.108.

4. Elimination of Lymphatic Filariasis India-2013-14, National Guidelines for Transmission Assessment Survey(For district level health Officials), National Vector Borne Disease Control Programme (NVBDCP), Gol publn. pp. 73. 